Voix et Images

voixetimages

\title{
Sur la piste américaine : le statut des références littéraires dans l'oeuvre de Jacques Poulin
}

\section{Jean-Pierre Lapointe}

Volume 15, numéro 1 (43), automne 1989

Jacques Poulin

URI : https://id.erudit.org/iderudit/200812ar

DOI : https://doi.org/10.7202/200812ar

Aller au sommaire du numéro

Éditeur(s)

Université du Québec à Montréal

ISSN

0318-9201 (imprimé)

1705-933X (numérique)

Découvrir la revue

\section{Citer cet article}

Lapointe, J.-P. (1989). Sur la piste américaine : le statut des références littéraires dans l'oeuvre de Jacques Poulin. Voix et Images, 15(1), 15-27. https://doi.org/10.7202/200812ar d'utilisation que vous pouvez consulter en ligne. 


\title{
Sur la piste américaine: le statut des références littéraires dans l'œuvre de Jacques Poulin
}

\author{
par Jean-Pierre Lapointe, université Trent
}

Moi, $j$ 'ai presque tout oublié ce que j'ai lu...
Teddy Bear ${ }^{1}$

Ce que l'on croit être un livre n'est la plupart du temps qu'une partie d'un autre livre plus vaste auquel plusieurs auteurs ont collaboré sans le savoir.

Jack Waterman 2

Les références à la littérature - et en particulier à la littérature américaine abondent dans l'œuvre de Jacques Poulin. D'un roman à l'autre, des noms d'écrivains, des titres de livres, surgissent au détour d'un dialogue ou dans un repli de la trame narrative, cortège insolite dont le défilé semble procéder d'une volonté systématique de l'auteur, mais dans quelle intention au juste? S'agit-il, tel qu'il le laisse entendre, d'une énumération innocente de quelques ouvrages ou auteurs de prédilection, réitérés par les personnages, sorte d'hommage naïf et spontané à des livres aimés? Ou ne faut-il pas chercher à y voir au contraire un réseau d'indices qui invite le lecteur à un repérage attentif, voire à un décodage rigoureux? En fait, le défilé d'écrivains ne commence qu'avec le second roman, car dans Mon cheval pour un royaume, hormis la citation de Rainer Maria Rilke en exergue, il n'y a aucune référence littéraire directe. En revanche, on y trouve des bribes, peut-être parodiques, qui évoquent Saint-Denys Garneau, Hubert Aquin et Alain Robbe-Grillet, au cœur d'une écriture plus proche du Nouveau Roman que d'une quelconque influence américaine.

Dans Jimmy, le récit est placé sous l'invocation explicite de J.D. Salinger, même s'il est surtout question d'Ernest Hemingway. Pas de référence à

1 Les Grandes Marées, p. 84. Dans les renvois subséquents, les romans de l'auteur seront désignés par les titres suivants: MCPR [Mon cheval pour un royaume, Montréal, Leméac, 1987 (Poche Québec littérature)]; J [Jimmy, Montréal, Stanké, 1985 (Québec 10/10)]; CBB [le Cour de la baleine bleue, Montréal, Éditions du Jour, 1979 (le Petit Jour)]; FBR [Faites de beaux rêves, Montréal, l'Actuelle, 1974]; GM [les Grandes Marées, Montréal, Leméac, 1978], VB [Volkswagen Blues, Montréal, Québec/Amérique, 1984 (Littérature d'Amérique)]; VC [le Vieux Chagrin, à paraître].

VB, p. 169. 
quiconque d'autre. Dans le Cœur de la baleine bleue, parmi des auteurs québécois et français, on retrouve J.D. Salinger, Henry Miller et Francis Scott Fitzgerald. Ce sont encore Salinger et Fitzgerald qui reviennent dans Faites de beaux rêves, tandis que Kurt Vonnegut et Ray Bradbury figurent dans les Grandes Marées. Enfin, Volkswagen Blues fait défiler John Irving, Jack Kerouac et quelques autres poètes beat, Jack London, William Faulkner, Mark Twain, Saul Bellow, Lawrence Ferlinghetti et, bien entendu, Hemingway. C'est dans ce roman que le protagoniste identifie nommément ses auteurs préférés:

Il avait ses auteurs favoris dont il avait lu tous les livres mais ces auteurs n'étaient pas nombreux: Hemingway, Réjean Ducharme, Gabrielle Roy, Salinger, Boris Vian, Brautigan et quelques autres. (VB, p. 42)

Cependant tous les auteurs ne jouissent pas du même statut, peu s'en faut! Hemingway, d'emblée le plus présent, est mentionné quinze fois dans trois romans ${ }^{3}$. Il devient même un quasi-personnage sous les traits de Papou dans Jimmy ${ }^{4}$, puis sous ceux d'un vagabond mythomane dans Volkswagen Blues. Saul Bellow et Lawrence Ferlinghetti accèdent, quant à eux, au statut de personnages vrais (figurants) puisqu'ils tiennent chacun un rôle dans Volkswagen Blues. Faulkner, Miller et Bradbury, par contre, n'ont droit qu'à une seule mention, même si ce dernier est cité concrètement dans les Grandes Marées (p. 49).

D'aucuns ont vu dans cette pratique référentielle une évocation des influences américaines, lesquelles seraient facilement reconnaissables dans le style et dans la thématique de l'œuvre de Poulin ${ }^{5}$. Cette affirmation mérite qu'on s'y arrête et qu'on la vérifie. Y a-t-il effectivement des influences reconnaissables? Dans quelle mesure et sous quelles formes les retrouve-t-on dans les récits de Poulin?

Il va sans dire que la problématique d'une telle enquête s'annonce aussi vaste que la méthodologie en serait laborieuse. La liste des écrivains nommés contient pas moins de quinze romanciers américains (avec une brève mention de trois poètes beat ${ }^{6}$ en plus d'auteurs français et québécois. Quant aux romans nommés ou cités, ils se chiffrent à huit ${ }^{7}$. À cela, il faut ajouter les ouvrages non littéraires, les monographies historiques, qui auraient pu aussi servir

3 Sur le rapport privilégié qu'entretient Jacques Poulin avec l'œuvre d'Emest Hemingway, voir «Entretien avec Jacques Poulin» dans le présent numéro de Voix \& images, p. 8-14.

4 [...] et tout d'un coup le vieux Hemingway lui-même, avec sa fameuse barbe grise, son fusil et tout, se met à descendre l'escalier à la place de Papou. (J, p. 87)

5 Jonathan Weiss, «Une lecture américaine de Volkswagen Blues», Études françaises, vol. XXI, no 3, hiver 1985-1986, p. 91.

6 Les romanciers Twain, London, Faulkner, Hemingway, Fitzgerald, Salinger, Miller, Brautigan, McCullers, Bellow, Bradbury, Ferlinghetti, Vonnegut, Kerouac, Irving, et les poètes Ginsberg, Orlovsky, Tom Wolfe.

7 The Old Man and the Sea, The Great Gatsby, The Adventures of Augie March, Humboldt's Gift, Cat's Craddle, The Catcher in the Rye, On the Road, Hotel New Hampshire. 
d'intertextes ${ }^{8}$. Corpus diversifié et quelque peu flou puisque la seule mention d'un écrivain ne permet pas de supposer que l'auteur québécois a lu son œuvre entière, ni même qu'il en ait subi quelque influence. À cet égard, il importe de traiter différemment les influences thématiques et les influences formelles, car si celles-ci transparaissent plus nettement dans la relation mimétique, les premières, en revanche, sont plus difficiles à attribuer. En effet, les thèmes d'un ouvrage littéraire sont rarement exclusifs à son seul auteur. Le plus souvent, ils sont le reflet des courants culturels qui traversent l'époque. On peut ainsi trouver dans la littérature américaine $\mathrm{du} \mathrm{XX}^{\mathrm{e}}$ siècle un certain nombre de thèmes communs à plusieurs écrivains d'une même génération. Par contre, le caractère formel d'une œuvre donnée est beaucoup plus particulier car, au-delà des techniques et des modes propres à la tradition ou à l'époque, la signature stylistique d'un écrivain est individuelle.

À première vue, certaines affinités thématiques des récits de Jacques Poulin avec la littérature américaine sautent aux yeux. Le mythe du paradis perdu, par exemple, si présent dans le roman américain depuis le $\mathrm{XIX}^{\mathrm{e}}$ siècle $^{9}$, forme le thème central des Grandes Marées puisque Teddy Bear et sa compagne Marie sont évincés de leur paradis terrestre (p. 34) par les représentants de la civilisation contemporaine envahissante. Dans Volkswagen Blues, au fil du récit se succèdent et se superposent les «paradis perdus» de l'Amérique autochtone, de l'Eldorado, de l'Amérique française, de la Prairie, de la Californie et autres plaies mal cicatrisées du grand rêve de l'Amérique (p. 100). Poulin définit ce rêve comme la réalisation d'un pays où tous les gens seraient libres et heureux (VB, p. 101). Dans l'univers de Poulin, bien que la quête universelle du bonheur soit soulevée à maintes reprises, les personnages ne sont pas engagés dans la recherche active du bonheur autant que dans l'attente (passive) du bonheur. Il ne s'agit pas davantage du bonheur collectif, de trouver le pays ou le paradis qui pourrait rendre tout le monde heureux. Le déroulement allégorique des Grandes Marées et les évocations répétées du «Grand rêve de l'Amérique» dans Faites de beaux rêves et Volkswagen Blues montrent clairement que le bonheur collectif est irréalisable.

Le bonheur est donc affaire d'individus. Il se trouve peut-être dans une patiente harmonie de l'individu avec son milieu, et peut-être aussi dans la capacité d'aimer. Au premier abord, le personnage principal chez Poulin n'est pas un homme heureux. Sa quiétude, son apparente disponibilité ne doivent pas nous tromper. Sa passivité, son manque d'agressivité, sa douceur (fausse) sont le signe, nous prévient-il, qu'il a du mal à vivre (VB, p. 211). C'est un inquiet, craintif par sentiment d'infériorité et méticuleux par insécurité. Mais il n'est pas malheureux non plus. Sa mélancolie est sereine, résignée, avec parfois plus qu'un soupçon d'ironie ${ }^{10}$. Jamais il ne s'apitoie. Ses moments de tristesse,

8 Notamment The Golden Dream de W. Chapman et The Oregon Trail Revisited de G. Franzwa.

9 Voir Jacques Cabau, la Prairie perdue, Paris, Seuil, 1966, p. 19.

10 Dans le Cour de la baleine bleue, la scène du départ d'Élise avec Bill (p. 123-140) laisse deviner la grande douleur qui étreint Noël, mais par réflexe d'autodéfense, il conserve un ton retenu, mesuré; son point de vue est axé sur les détails ironiques plutôt que sur son amertume. 
comme ses joies, sont éphémères. S'il s'interroge parfois sur le bonheur, c'est pour admettre aussitôt que le bonheur est indéfinissable, et donc insaisissable: Comment fait-on pour savoir si on est heureux ou non? demande Teddy Bear au patron (GM, p. 91).

Chaque roman toutefois est traversé par un personnage qui semble plus doué, instinctivement, pour le bonheur. Ce sont des êtres rares, des femmes exclusivement, qui réunissent confiance en soi, connaissance de soi, chaleur et générosité, mais qui restent farouchement libres et indépendantes: Charlie, Limoilou, Marie, Pitsémine. On les sent habitées d'une sérénité, d'une grâce toute proche du bonheur, qui restera néanmoins inaccessible à leur compagnon mâle parce que le bonheur, comme l'amour, ne se partage pas.

Est-il besoin de rappeler que la difficulté d'aimer, sujet universel s'il en est un, occupe une place dominante dans la littérature américaine qui fut, et reste encore, une littérature sentimentale. Les exemples d'amours malheureuses, contrées par les événements ou les situations, sont légion, mais ce qu'il faut retenir, c'est le thème de l'amour inaccompli, échoué par défaut d'âme, échappé par inconscience. Le climat moral d'une Amérique longtemps puritaine explique en partie la pudeur avec laquelle les écrivains traitent des passions. À cause de cela sans doute, il y a au fond de l'idéal américain un mythe de la chasteté mâle qui s'exprime dans des images insistantes de virilité. Les liens profonds du cœur se lient entre hommes, mais restent inavoués ${ }^{11}$. La femme, quand elle n'est pas l'incarnation du Mal ${ }^{12}$, se présente sous des traits qui l'apparentent à l'homme: cheveux courts, tenue masculine, comportements libres, téméraires ${ }^{13}$. Chez Poulin, la femme n'est jamais le Mal. Même quand Élise le trompe et le trahit, l'homme ne lui en fait pas reproche, s'interdit de la juger. Mais combien de femmes-garçons traversent ce roman? Depuis les adolescentes à la sexualité indistincte des premiers récits jusqu'à la Grande Sauterelle qui connaît les secrets de la mécanique automobile et qui se travestit en garçon, les personnages féminins sont caractérisés principalement par leur refus des passions. Chaleureuses, compréhensives, maternelles parfois, et à l'occasion spontanément sexuelles, ces femmes-enfants ${ }^{14}$ n'exigent rien de l'homme que sa douceur et ne lui offrent aucun attachement en retour. Comme si le prix du bonheur, de la quiétude, de l'harmonie, était le refus de l'amour. Ou peut-être parce que l'amour, en définitive, est tout aussi inconnu et insaisissable que le bonheur.

De tous les paradis perdus, cependant, c'est l'enfance dont la perte est la plus troublante et la nostalgie la plus vive. Depuis Jimmy, l'œuvre de Poulin est hantée par le mystère de l'enfance. Dans ce roman, le narrateur-enfant assiste inconscient d'abord, impuissant ensuite, à la dissolution de son enfance. En dépit des remparts fantaisistes et ludiques qu'il érige instinctivement, obsessivement,

11 Jacques Cabau, op. cit., p. 20.

12 Par exemple The Scarlet Letter de Nathaniel Hawthorne.

13 C'est souvent le cas chez Hemingway, Fitzgerald, Miller.

14 Retenons aussi les belles pages qui racontent la tendre et innocente camaraderie de Jimmy avec Mary dans Jimmy. 
la dure réalité adulte envahit impitoyablement son paradis et le submerge. Le cri de détresse qui clôt le récit: BESOIN DE TENDRESSE!, over (J, p. 158), va se répercuter dans tous les récits subséquents, telle une sourde et douloureuse révolte contre la mutilation de l'innocence. Le personnage central ne se résigne pas à la nécessité de quitter l'enfance. Dans le Cœur de la baleine bleue, le plus ouvertement introspectif des romans de Poulin, le narrateur mesure le risque de s'égarer dans l'enfance:

Quand vous voyagez à l'intérieur de vous-même, les courants vous entraînent fatalement vers votre enfance et parmi les paysages anciens de la mémoire vous risquez fort de retrouver des souvenirs qui vous feront perdre le chemin du retour. (CBB, p. 147)

N'est-ce pas justement pour retrouver le chemin du retour que Jack Waterman part à la recherche de Théo dans Volkswagen Blues? À quarante ans passés, Jack pourrait dire, comme Noël quinze ans plus tôt: [...] mon enfance $n^{\prime}$ est pas vraiment terminée (CBB, p. 23).

Car dans l'intervalle, le personnage central est resté comme détaché du présent: son étrange passivité, sa douceur terne et mélancolique, son indifférence désabusée, masquent effectivement les souvenirs d'une enfance pourtant plus ardente, laquelle s'éveille à l'occasion en de belles images complexes de l'eau, du feu, de la maison ${ }^{15}$. C'est de son enfance, et non de son existence adulte, dont Jack parle à Pitsémine pour se révéler pudiquement à elle lors des premières confidences, comme si son identité était restée depuis longtemps à l'état virtuel, ou qu'elle ait été refoulée, sous l'assaut des désillusions de la vie adulte, vers cette seule terre ferme. Même son nom, son identité d'écrivain, lui vient d'une fantaisie enfantine de son frère (VB, p. 14, 42).

Or c'est en remontant dans les deux sens le cours du temps qui le sépare de l'enfance, c'est-à-dire de son frère, que Jack parviendra à se libérer peut-être du poids de l'enfance. Ayant enfin démythifié Théo, ce personnage moitié vrai, moitié inventé (VB, p. 137), Jack comprend soudainement qu'il ne l'aimait peut-être pas vraiment: Peut-être que j'aimais seulement l' image que je m'étais faite de lui. (VB, p. 289) Cela vaudrait-il pour l'enfance également? Quand, au moment de leur séparation, la Grande Sauterelle, citant Daniel Boone, lui rappelle que malgré les tourbillons la vie va de l'avant, Jack ne fait aucun commentaire, et pour la première fois dans l'œuvre, le récit s'arrête non sur la mort ou l'échec et la solitude, mais sur un sourire optimiste, signe certain d'une réconciliation avec le présent (VB, p. 289).

Le mirage de l'enfance qui fascine les personnages de Poulin, c'est la fuite en arrière vers la sécurité des origines, et c'est surtout le regret de l'innocence perdue. Ce thème, on le sait, est un leitmotiv important de la littérature américaine moderne. Parmi les auteurs de prédilection de Poulin, Hemingway (The

15 Ces images ne sont pas sans rappeler Bachelard que Poulin a sans doute lu. Voir le Cœur de la baleine bleue, p. 29. 
Old Man and the Sea), Salinger (The Catcher in the Rye), Vonnegut (Cat's Craddle) et, bien entendu, Jack Kerouac (Visions of Gerard, Doctor Sax), illustrent dans l'après-guerre l'emprise durable du mythe de l'enfance sur la conscience américaine. Mais l'importance du mythe était déjà manifeste dans des classiques comme Moby Dick, Huckleberry Finn et les récits de Fenimore Cooper, car l'idéal américain d'énergie, de liberté, de justice et de bonheur s'y exprimait en des personnages qui ont la droiture, la naïveté et l'innocence des enfants ${ }^{16}$. C'est le même idéal qui guide la perspective du roman réaliste de Sinclair à Steinbeck. Il faudra les angoisses et les désillusions successives occasionnées par la Crise, la Deuxième Guerre, la bombe atomique et, plus près de nous, par la lutte pour les droits civiques et par la guerre du Viêt-nam, pour que commence à s'effriter la foi commune en l'innocence. Un écrivain que cite Poulin, Carson McCullers, dans The Heart Is a Lonely Hunter, a su capter la détresse de la sensibilité moderne, l'échec de l'amour réciproque, la solitude ultime de chacun au cour de la foule anonyme.

Le mythe de l'innocence, issu en vérité des origines puritaines de la société américaine, $s$ 'est perpétué dans une vision morale et sentimentale du héros solitaire, qui à elle seule sous-tend de larges pans de la littérature populaire, du cinéma et de la bande dessinée. Le héros s'impose à notre attention par son adresse, son courage, mais c'est sa lucidité qui commande le respect, c'est-àdire sa faculté de définir des tâches nécessaires et de se donner une ligne de conduite qui garantisse leur réalisation en dépit des imprévus. Le culte du héros s'incarne dans l'œuvre de Poulin sous une multitude de traits. Au premier plan, Théo, le frère aventurier peu à peu mythifié par l'absence et le recul des souvenirs ${ }^{17}$. Entre autres fonctions, il représente pour le narrateur la sagesse née du savoir et de l'expérience. Théo est un mentor parce que depuis l'enfance, il est l'initiateur aux secrets du monde. Mais c'est un modèle inatteignable. Au second plan, une pléiade de héros populaires, sportifs (Maurice Richard, Jimmy Clark), historiques (Étienne Brûlé, Buffalo Bill) et littéraires (Hemingway) que la légende nous présente assurés, audacieux, volontaires ${ }^{18}$. Incarnation ironique, en contrepartie, que celle du narrateur anti-héros, passif, timide et craintif. Cependant, l'écart rétrécit au fil des récits, car l'image du héros se trouble et s'estompe, brouillée par le doute et l'ambiguïté historique pour enfin disparaître au moment où le narrateur atteint à une nouvelle lucidité qui lui montre la voie de l'autonomie et de la quiétude. Don't talk to me about heroes (VB, p. 279), réplique un peu impatiemment Jack à une vieille chanteuse de rue excentrique, échouée à San Francisco, qui est portée à mythifier le passé. La chute de ses héros est la conséquence inévitable de sa propre maturation. Comme il l'a compris en suivant la Piste de l'Oregon, les héros de l'histoire, ce sont les gens

16 Voir Leslie Fielder, An End to Innocence, Boston, Beacon Press, 1957, p. 144 et ss.

17 Acteur dominant dans Faites de beaux rêves, Théo devient présence spirituelle dans les Grandes Marées et objet mythifié de la quête dans Volkswagen Blues.

18 Le héros ne doute jamais longtemps de lui-même: Théo [...] était absolument convaincu qu'il était capable de faire tout ce qu'il voulait. (VB, p. 137) 
ordinaires qui affrontent les plus grandes difficultés parce qu'ils ont foi en un monde meilleur.

Vestige de 1'histoire des explorations et des migrations transcontinentales, le thème du mouvement, du voyage, fonde lui aussi la littérature américaine depuis Fenimore Cooper. À la recherche du Paradis perdu ou dans la foulée du héros évanescent, le voyage devient errance déjà chez Mark Twain, exil avec la «lost generation» (Hemingway, Fitzgerald) et atteint son apogée anarchique dans la révolte beat avec Jack Kerouac, Burroughs et compagnie. Préfiguré par Théo, dont Jack Waterman nous dit qu'il est la partie de lui-même qui a oublié de vivre (VB, p. 137), le goût de l'errance ne gagne le narrateur que très lentement. Sédentaire, presque casanier dans les premiers récits, malgré le temps passé à errer dans les rues du Vieux-Québec (Mon cheval pour un royaume, le Cœur de la baleine bleue), le narrateur est successivement sollicité par l'appel du large (Jimmy), par les Etats-Unis et l'Eldorado (Faites de beaux rêves, les Grandes Marées), par la Piste de l'Oregon (les Grandes Marées) pour enfin s'abandonner à ce besoin d'errance (Volkswagen Blues).

Il est significatif que cette errance, qui emprunte le parcours des explorations et des migrations dans Volkswagen Blues, ne s'accomplit pas uniquement au gré de la recherche de Théo puisqu'elle se double en même temps d'un pèlerinage à plusieurs monuments de l'histoire littéraire américaine. C'est à Detroit que l'auteur pénètre l'Amérique états-unienne. Ses lointaines origines françaises réduites à son seul nom, cette ville, plus que toute autre, symbolise depuis longtemps l'idéal américain de la technologie au service des masses. Mais la murale de Diego Rivera que les voyageurs contemplent au Detroit Institute of Arts (VB, p. 92-93) expose l'échec accablant de ce rêve, tout comme l'épisode de la traversée avortée du parc signale brutalement la régression de la civilisation urbaine, devenue 1'habitat d'une faune meurtrière. C'est le lendemain, à Chicago, que le narrateur établit la jonction entre civilisation et littérature. Chicago est une ville-miroir, une ville «littéraire», à la fois sujet et berceau du roman réaliste, de la littérature comme conscience sociale (Dreiser, Anderson, Norris, Sinclair), de la littérature engagée (Sandburg, Algren, Richard Wright). Tout près, à Oak Park, naquit Hemingway. Sitôt arrivés, Jack Waterman et la Grande Sauterelle tiendront à visiter la galerie des French Impressionnists au Art Institute (VB, p. 105), le lieu même où le jeune Hemingway découvrait Monet, Manet et surtout Cézanne dont la technique picturale allait influencer si radicalement son style ${ }^{19}$.

La rencontre de Saul Bellow survenue le même jour est doublement significative. L'auteur des Aventures d'Augie March, figure dominante du roman américain de l'après-guerre, est, par ses préoccupations intellectuelles, l'un des plus européens des romanciers américains modernes ${ }^{20}$, un moraliste

19 Sheldon Grebstein, Hemingway's Craft, Carbondale, Southem Illinois University Press, 1973, p. 162.

20 Frank D. McConnell, Four Postwar American Novelists, Chicago, The University of Chicago Press, 1977, p. 2. 
qui veut découvrir cette terra incognita (VB, p. 111) de l'Amérique humaine à la mesure de l'individu, au sein d'une civilisation édifiée sur la liberté et devenue sauvage, violente, absurde, apocalyptique. L'appel de l'Amérique a toujours été ambivalent chez Poulin. Autant il s'alimente à cette culture, à ses créations culturelles (livres, films, musique) et à ses produits de consommation, autant il témoigne d'un certain malaise devant l'énergie brouillonne, la mentalité agressive qui fusent de partout: [...] l'espoir fou qu' on peut tout faire de force (CBB, p. 62). L'envers sinistre de l'énergie, c'est la violence dont Jack découvre partout les traces lors de son exploration de l'Amérique historique: On dirait que toute l'Amérique a été construite sur la violence. (VB, p. 129) Bellow rappelle à ses deux admirateurs francophones que Chicago fut jadis une ville immorale et brutale (wicked, rough) et que la violence y coexiste aujourd'hui avec les affaires et la culture. Creuset de la société de consommation (Zenith TV, Wrigley's, McDonald's), la métropole du Mid-West est l'illustration du paradoxe existentiel de la civilisation américaine, qu'on retrouve au centre de l'œuvre de Saul Bellow: la recherche du mieux-être collectif par le progrès matériel et le perfectionnement de l'institution sociale ont atrophié la conscience de soi et aboli la primauté de l'individu (ce thème est magistralement exprimé dans la murale de Diego Rivera). Sceptique par besoin de lucidité, apôtre irréductible du droit à la dissidence, Bellow croit néanmoins que l'antidote, c'est la fraternité, l'identité à l'espèce, moyen nécessaire de revivifier les préceptes et les valeurs de la civilisation occidentale ${ }^{21}$. C'est pourquoi la réplique qu'il lance à Jack: Quand vous cherchez votre frère, vous cherchez tout le monde, tout en élargissant dans l'immédiat les horizons de la quête de Jack, dépasse ce moment et met en relief un thème qui est le commun dénominateur des romans de Jacques Poulin depuis Jimmy: celui de la fraternité, de la chaleur humaine. Effectivement, dans leurs contacts avec les Américains, Jack et son amie ne rencontrent le plus souvent que bienveillance, générosité et fraternité: l'Amérique humaine dont parle Bellow.

En quittant Chicago, les deux voyageurs se dirigent vers Saint Louis pour y retrouver la trace de Théo, mais, curieusement, au lieu de piquer par le plus court chemin vers le Sud-Ouest, ils empruntent d'abord la route 80 qui les conduit vers l'Ouest et les amène à Davenport, Idaho, où ils découvrent le Mississipi d'abord en flairant son odeur à distance: C'était une odeur humide et accablante, épaisse et comme un peu vaseuse [...] (VB, p. 117). Cette scène rappelle distinctement le parcours emprunté par Jack Kerouac dans On the Road, qui lui aussi roule de Chicago à Davenport et découvre le Mississipi à son odeur: [...] with its big rank smell that smells like the raw body of America itself ${ }^{22}$. Jack croisera de nouveau les traces de Kerouac à San Francisco. Même sans ces rappels explicites, Volkswagen Blues ferait penser à la célèbre odyssée de l'écrivain canuck. S'agitil d'influence, de parodie? Tout au plus d'un clin d'œil au lecteur car, hormis le motif du voyage transcontinental, les deux textes ne sont pas vraiment apparentés.

21 Ibid., p. 10.

22 Jack Kerouac, On the Road, London, Penguin Books, 1988, p. 17-18. 
L'errance de Sal Paradise est frénétique, carnavalesque, irrésistiblement entraînée par un besoin démentiel d'absolu: [...] the only people for me are the mad ones, the ones who are mad to live, mad to talk, mad to be saved, desirous of everything at the same time ${ }^{23}$. Le voyage de Jack Waterman, au contraire, aboutit à une calme prise en charge d'un moi purgé de ses angoisses, qui comprend - et accepte sans amertume et sans révolte - sa place dans l'ordre des choses.

Longeant le Mississipi légendaire, mythique et qui se confond avec l'âme de l'Amérique (VB, p. 118), Jack évoque Mark Twain et Faulkner du même souffle qu'il énumère les images des esclaves noirs, des bayous et du jazz. L'impression laissée est celle d'une Amérique lointaine, exotique. L'hommage distant qu'il rend aux deux grands écrivains sudistes s'explique sans doute par le peu d'ascendant qu'ils ont eu sur Poulin directement. Pourtant, le réalisme linguistique de Twain, qui a engendré la langue littéraire américaine en la distanciant de l'anglais littéraire britannique, n'est pas si différent du style naturel, parlé, concret que Poulin s'efforce depuis longtemps de pratiquer luimême à l'instar d'Hemingway qui lui, reconnaît sans hésiter le rôle initiateur de Mark Twain 24. Pour ce qui est de Faulkner, la distance n'est que logique, puisqu'il est clair que son univers à la fois gothique et romantique est aux antipodes du monde de Poulin. Aussi la descente vers le Sud s'interrompt-elle à Saint Louis, et c'est vers l'Ouest, et ultimement vers la Californie, que se poursuit ce pèlerinage. Ici l'histoire se substitue momentanément à la fiction. La traversée de la Prairie s'accomplira suivant la monographie de Franzwa, The Oregon Trail Revisited, plutôt qu'au moyen de jalons littéraires tel qu'auparavant. Comme si la fiction ne pouvait rendre justice à l'histoire ni accréditer adéquatement le courage de ces héros anonymes, ces gens ordinaires (VB, p. 133) que furent les pionniers de l'Ouest. Jack puise aussi volontiers dans l'histoire orale relatée par les divers informateurs rencontrés, pour reconstituer le tableau véridique de l'histoire.

Quand la figure de l'écrivain reparaît, c'est sous les traits d'un mythomaneconteur, qui se prend pour Hemingway (VB, p. 237), puis par la mention de la ville de Ketchum, Idaho, où Hemingway toucha le fond de son angoisse avant de se suicider. Deux images qui suggèrent respectivement l'imposture et l'échec et sont à mettre en parallèle avec la démythification du héros.

Bref le cheminement thématique de l'œuvre poulinienne emprunte un parcours qui traverse le paysage familier de la fiction américaine. L'auteur n'hésite pas à affirmer lui-même cette coöncidence lorsqu'il dit: $M o i$, ce que $j$ 'aime le mieux ce sont les romanciers américains; je m'y sens chez nous. ${ }^{25}$ Reste à voir de quelle nature est cette coïncidence. Est-elle une expression mimétique spontanée de la sensibilité littéraire américaine de l'écrivain québécois ou s'opère-t-elle au moyen d'un mécanisme parodique dont le texte d'arrivée est délibérément distancié de sa source?

23 Ibid., p. 11.

24 Jacques Cabau, op. cit., p. 142.

25 Entrevue avec Jean Royer, le Devoir, 29 avril 1978, p. 33. 
C'est au niveau formel, structurel et stylistique que l'influence, si elle existe, serait plus facilement mesurable. Or qu'en est-il? Y a-t-il imitation? Volonté de parodier? Certes on note des collages, des citations, mais on trouve plus difficilement des pastiches, des fragments parodiques reconnaissables, car la barrière linguistique (le passage de l'anglais au français) masque la ressemblance. Si parenté il y a, elle résiderait plutôt dans une notion de l'écriture que dans l'imitation textuelle proprement dite.

La critique a largement commenté le dépouillement progressif du style de Poulin, depuis l'écriture sinueuse et cahin-caha de Mon cheval pour un royaume ${ }^{26}$, orientée vers le Nouveau Roman, jusqu'à l'épurement absolu, la légèreté limpide de Volkswagen Blues ${ }^{27}$. De tous les écrivains américains, celui que Poulin rappelle le plus, c'est bien sûr Hemingway dont le nom et la présence refont surface un peu partout à travers l'œuvre. Le style d'Hemingway, tout en ellipse, qui prétend à l'objectivité du procès-verbal, à la préhension directe du réel, par l'extérieur, par le refus de toute médiation, pourrait décrire le but de l'évolution stylistique de Jacques Poulin. Dès le moment où la voix narrative passe de la première personne à la troisième ${ }^{28}$, l'optique narrative se distance de son objet et le silence, le non-dit, tout autant sinon plus que le dit, devient le lieu du communicable. Cela fait penser à la célèbre «théorie de l'iceberg» mise en valeur par Hemingway, selon laquelle le récit doit laisser entendre beaucoup plus qu'il ne montre ${ }^{29}$. La description concrète et scrupuleusement objective des lieux, des objets et des gestes - la kinetographic fallacy ${ }^{30}$ - est le fondement de l'esthétique d'Hemingway. Il cherche à communiquer sans la décrire la relation précise entre ce qu'il voit et ce qu'il ressent ${ }^{31}$. De même, les dialogues doivent être directs; l'auteur ne se permet aucune intervention. La phrase du maitre artisan américain est caractérisée par sa brièveté, sa composition linéaire à la voix active, son débit idéalement monosyllabique, quasi incantatoire ${ }^{32}$. Peu d'adjectifs et d'adverbes, une constante retenue dans l'emploi de tout ce qui pourrait agresser le lecteur, la couleur par exemple 33 . Point n'est besoin d'une analyse stylistique bien poussée pour faire ressortir les ressemblances du style de Poulin avec celui d'Hemingway.

26 Jean Ethier-Blais, «Mon cheval pour un royaume», le Devoir, 30 juin 1967, p. 15.

27 Régis Tremblay, «Déshabiller la phrase de ses adjectifs», le Soleil, 7 juillet 1984 , p. D2.

28 Faites de beaux rêves, 1974. Les trois premiers romans sont écrits à la première personne, les trois suivants à la troisième.

29 I always try to write on the principle of the iceberg. There is seven eights of it under water for every part that shows. Anything you know you can eliminate and it only strenghtens your iceberg. Cité par F.J. Svoboda, Hemingway \& The Sun Also Rises: The Crafting of a Style, Lawrence, University Press of Kansas, 1983, p. 19; The story carries the conviction it means more than it directly says, Carlos Baker, Hemingway: The Writer as Artist, Fourth Edition, Princeton, Princeton University Press, 1972, p. 322.

30 Carlos Baker, op. cit., p. 63.

31 Ibid., p. 85.

32 Sheldon Grebstein, op. cit., p. 133.

33 Carlos Baker, op. cit., p. 51. 
Phrases simples et dépouillées, retenue, objectivité. Comme Hemingway, Poulin a voulu faire l'expérience de diverses voix narratives. Hemingway pouvait passer avec une égale maîtrise de la première à la troisième personne et semblait préférer l'usage de cette dernière à des fins de détachement objectif, même si elle privait la narration de cette qualité d'authenticité de l'expérience vécue ${ }^{34}$. Jacques Poulin avoue que ce problème de la voix narrative est pour lui le plus sérieux, qu'il n'en est pas de plus difficile à résoudre ${ }^{35}$. Ajoutons à cela le souci, chez Poulin comme chez Hemingway, de la description détaillée des lieux, des objets, des actions, et l'on comprendra que l'admiration que Poulin voue candidement à son idole lui suggère une pratique stylistique, voire une conception de l'écriture, qui s'inspire beaucoup du modèle. Cependant la phrase de Poulin n'apparaît pas aussi sèche, aussi froide, que celle d'Hemingway. Même dans le dépouillement le plus absolu, passent une douceur, une sensibilité qui s'insèrent dans le tissu syntaxique de la phrase, comme dans le passage suivant où l'auteur, dans un style frugal et impersonnel, nous laisse néanmoins sentir l'inquiétude et la tristesse qui gagnent les deux personnages à l'idée que leur chat s'est perdu:

Jack la rejoignit. Ils franchirent la clôture et marchèrent ensemble dans le champ; le petit chat n'était pas là. Ils se mirent à faire le tour de rues voisines; ils examinaient les perrons et les galeries et, quand il n'y avait pas de monde, ils inspectaient les allées et les fonds de cour, mais ce fut en vain. (VB, p. 152)

La question des schèmes structuraux est plus épineuse. Jacques Poulin semble pointer du doigt des pistes à suivre. Aussi, avoue-t-il au lecteur, l'écriture de Jimmy a été amorcée par la lecture de The Catcher in the Rye, car c'est à ce moment qu'il a eu l'idée d'utiliser un jeune garçon comme narrateur, ce qui a donné au roman un ton spécial (J, p. 161). La langue de Jimmy n'est pas sans rappeler, en effet, celle de Holden Caufield. Mêmes tics verbaux: Honestly jure souvent Holden dont les phrases sont aussi ponctuées de I mean, and all et or something. Le narrateur de Jimmy, quant à lui, répète souvent pour être honnête et se rabat à l'occasion sur des tournures passepartout telles que je veux dire et des choses comme ça. Tics idiomatiques propres à l'enfance, suppose-t-on. Mais à l'étude, on s'aperçoit que de telles tournures sont beaucoup moins fréquentes chez Poulin et que la langue de Jimmy est nettement plus précise, plus articulée, que celle de Holden, comme on peut le voir en comparant les deux passages descriptifs suivants:

Subitement, en face de nous, c'est comme un phare qui s'allume et s'éteint: la porte de notre chalet s'est ouverte, refermée tout de suite, et tu devines une mince silhouette blanche qui descend l'échelle de fer. (J, p. 135)

34 Sheldon Grebstein, op. cit., p. 53-55.

35 Voir «Entretien avec Jacques Poulin» dans le présent numéro de Voix \& images, p. 14. 
It was lousy in the park. It wasn't too cold, but the sun still wasn't out, and there didn't look like there was anything in the park except dog crap and globs of spit and cigar buts from old men [...]. ${ }^{36}$

Pourtant Holden a seize ans tandis que Jimmy n'en a que onze. Au-delà des emprunts superficiels avoués, les structures respectives des deux récits sont bien différentes. Holden raconte une fugue, une dérive dans un territoire inconnu (New York) et par son discours truculent, ironique et mordant, expose une sensibilité écorchée et déchaîne sa révolte contre l'imposture du monde adulte. Jimmy, de son côté, reste au centre d'une famille en détresse et ultimement en voie de dissolution. Il s'accroche à l'enfance, use de son imagination pour poétiser le réel et non pour le fuir. La révolte gronde mais n'éclate que vers la fin dans un cri de désarroi.

La référence à Cat's Craddle est d'un autre ordre. Interrogé par Marie, Teddy Bear dit ne pas connaître ce roman de Vonnegut, dont il a peine à saisir le titre, le confondant avec SCRABBLE (GM, p. 96). Ce désaveu explicite est-il un écran que Jacques Poulin dresse pour camoufler un rapport quelconque des Grandes Marées avec ce roman? Certes, ni le récit ni le sujet ne se touchent vraiment. Vonnegut écrit à la première personne tandis que Poulin, depuis le roman précédent, a opté pour la troisième personne. Le style du satiriste américain va et vient entre le dépouillement narratif et la description surchargée d'affectivité: We approached the light and found that it was sunshine to a fantastic little country built on plywood, an island as perfectly rectangular as a township in Kansas. ${ }^{37}$ Seule ressemblance structurale possible: le découpage des deux récits en chapitres très brefs et portant chacun un titre, encore que les chapitres de Cat's Craddle soient beaucoup plus brefs et plus nombreux que les chapitres des Grandes Marées. La structure narrative du roman de Vonnegut est linéaire, chaque chapitre s'arrête au point saillant de l'épisode, dont l'action s'enchaîne au chapitre suivant pàr une explication qui réduit le fait saillant à une banalité sans conséquence. Le récit tout entier est cimenté par un motif de causalité imprévisible, le narrateur protagoniste ne sachant pas d'une page à l'autre ce que lui réserve le prochain événement, la prochaine rencontre, conformément en cela à la philosophie bokononiste ${ }^{38}$ qui sous-tend le récit et selon laquelle l'individu n'a aucun moyen de comprendre la fatalité qui régit sa vie. Dans ce long conte satirique, tissé d'humour noir, l'on retrace la tentative par deux aventuriers de créer dans une île des Caraibes un paradis utopique destiné à assurer à tous ses habitants le bonheur le plus complet ${ }^{39}$. On reconnaîtra à quelques détails près le sujet des Grandes Marées. Mais alors que le paradis de Vonnegut, né de la supercherie et du mensonge, s'effondre dans la mégalomanie et la démence, la société utopique qu'on veut construire à l'île Madame, au cœur du Saint-Laurent, est congestionnée par la bonne volonté et

36 J.D. Salinger, The Catcher in the Rye, New York, Bantam Books, 1986, p. 118.

37 Kurt Vonnegut, Cat's Craddle, New York, Laurel, 1988, p. 56-57.

38 Ibid., p. 11 et ss.

39 Ibid., p. 90. 
les valeurs irréversibles de la civilisation moderne. Le récit de Vonnegut est une satire caricaturale qui se situe aux confins du fantastique tandis que le récit de Poulin tient plus de la fable poétique que de la satire.

Au terme de ces rapprochements sans doute abrupts et de ces comparaisons forcément sommaires, que faut-il conclure? Loin de voiler des influences possibles, Jacques Poulin prend plaisir à afficher ses auteurs préférés, aiguisant par le fait même la curiosité du lecteur, le mettant au défi de dépister des liens possibles. L'hypothèse d'un réseau quelque peu énigmatique d'indices littéraires à décoder n'est pas à retenir puisque même si tous ces écrivains ont eu, à des degrés divers, quelque ascendant sur Poulin, rien ne permet de proposer qu'ils forment un noyau, un ensemble, c'est-à-dire qu'ils auraient entre eux quelque parenté. Reste donc la question des influences particulières et unilatérales. Il est assez clair qu'un nom domine tous les autres: Hemingway. L'influence thématique de ce dernier n'est pas très étendue. Poulin n'a jusqu'à présent parlé ni de guerre, ni de chasse, ni de pêche à l'espadon. Mais l'influence stylistique d'Hemingway, sa notion instinctive de l'esthétique littéraire, trouvent de nombreux échos dans la conception que se fait Poulin de l'écriture. Y a-t-il imitation? Pas dans le sens strict, car le style de Poulin a évolué progressivement d'un livre à l'autre vers une forme de minimalisme qui doit moins à Hemingway qu'à la génération suivante représentée par Brautigan et Carver ${ }^{40}$. D'autant plus que le style d'Hemingway est marié à une action toujours dramatique tandis que celui de Poulin s'appuie sur une action sans éclat, parce que quotidienne et habituellement banale. La notion résolument anti-intellectuelle que se faisait Hemingway du métier littéraire pourrait expliquer elle aussi l'ascendant qu'il a eu sur son admirateur québécois. On sait que Poulin n'a jamais encore fait place à l'idéologie dans son œuvre, et qu'à l'occasion, il s'est moqué gentiment du monde intellectuel 41 . Or la présence, dans Volkswagen Blues, de Saul Bellow, romancier idéologique, pourrait signaler la tentation du roman à idées, tentation que Poulin avouait déjà dans une entrevue 42 .

Les autres écrivains auxquels il fait référence n'ont certes pas eu le même impact qu'Hemingway. Leur passage dans les récits de Poulin n'est pas la preuve d'une influence, mais plutôt qu'ils ont, chacun de leur côté, défini et occupé une parcelle du territoire littéraire américain que Jacques Poulin revendique comme le sien ${ }^{43}$.

40 Voir «Entretien avec Jacques Poulin» dans le présent numéro, p. 11.

41 On pense au professeur Mocassin dans les Grandes Marées et à cette remarque ironique dans Mon cheval pour un royaume: $[. .$.$] un intellectuel ça pense que$ rien c'est quelque chose alors les idées mécaniquement embrayent l'une dans l'autre [...]. (p. 61)

42 Voir Hélène de Billy, «Une Amérique panoramique sur la pointe des pieds», le Devoir, 19 mai 1984, p. 25.

43 Je suis un écrivain de l'Amérique qui écrit en langue française, J.P. Soulié, «Jacques Poulin après Volkswagen Blues: d'abord vivre une histoire d'amour», la Presse, 7 juillet 1984, p. B-3. 